\title{
Gravitação escalar: inconsistência das órbitas planetárias
}

\author{
Scalar gravitation: inconsistency of planetary orbits
}

\author{
T. G. Ribeiro*
}

Instituto de Ciências Exatas, Universidade Federal de Juiz de Fora, Juiz de Fora, MG, Brasil

Recebido em 11 de abril, 2016. Revisado em 12 de julho, 2016. Aceito em 31 de julho, 2016

Este trabalho estuda o movimento planetário na gravitação relativística descrita por um campo escalar. Em particular, com base na resolução de um exercício da Ref. [1], mostra-se que o movimento de precessão do periélio é inconsistente com o resultado previsto pela relatividade geral e com os dados experimentais observados.

Palavras-chave: gravitação escalar, campo escalar, órbitas planetárias.

This work studies the planetary motion in relativistic gravitation described by a scalar field. In particular, based on the resolution of an exercise of Ref. [1, it shows that the precession motion of the perihelion is inconsistent with the result predicted by general relativity and the observed experimental data.

Keywords: scalar gravitation, scalar field, planetary orbits.

\section{Introdução}

Atualmente o campo gravitacional é descrito no contexto da relatividade especial pela teoria geral da relatividade de Einstein. Nesta teoria a gravitação ganha um caráter puramente geométrico e é vista como a curvatura do espaço-tempo em resposta a determinado conteúdo de matéria e energia. No entanto, embora a relatividade geral seja aceita nos dias de hoje com base nas suas verificações experimentais, inicialmente a conexão entre gravitação e geometria do espaço-tempo não está evidente quando se trata a questão de incluir o campo gravitacional de Newton no contexto relativístico.

No presente artigo considera-se a possibilidade de generalizar o campo newtoniano por meio de um campo $\phi\left(x^{\mu}\right)$ que é um escalar do ponto de vista da relatividade especial. Os resultados teóricos, que em princípio não descartam uma possível gravitação escalar, são aplicados ao estudo do movimento planetário com a finalidade de solucionar o exercício 3.1 da Ref. 11. A solução encontrada deixa evidente que o resultado dessa teoria de gravitação para as órbitas planetárias é inconsistente com os dados

*Endereço de correspondência: tiaggribeiro@gmail.com experimentais e as previsões da relatividade geral. Levando, inclusive, a um movimento de retrocesso do periélio enquanto o que realmente se observa é um movimento de avanço.

\section{O espaço de Minkowski}

Com o advento da relatividade especial de Einstein a partir de 1905, as leis da física, válidas em todos os sistemas inerciais de referência, deixam de ser invariantes do ponto de vista das transformações de Galileu e passam a ser invariantes perante as transformações de Lorentz. Em particular, a velocidade da luz, c, é uma constante universal, ou seja, uma lei física válida em todos os sistemas de referenciais inerciais.

Como um caso particular das transformações de Lorentz, sejam $K$ e $K^{\prime}$ dois referenciais inerciais com eixos paralelos e origens que se coincidem no instante inicial. $K^{\prime}$ move-se em relação a $K$ com uma velocidade constante $v$ paralela ao eixo $x$. O referencial $K$ atribui coordenadas $(x, y, z, t)$ a um determinado evento ocorrido no instante de tempo $t$ e no ponto do espaço tridimensional caracterizado por $(x, y, z)$, enquanto que, para esse mesmo evento, 
$K^{\prime}$ atribui coordenadas $\left(x^{\prime}, y^{\prime}, z^{\prime}, t^{\prime}\right)$. Esses dois conjuntos de coordenadas estão relacionados por uma transformação de Lorentz, na forma [2]

$$
\begin{aligned}
x^{\prime} & =\gamma(x-v t) \\
y^{\prime} & =y \\
z^{\prime} & =z \\
t^{\prime} & =\gamma\left(t-\frac{v x}{c^{2}}\right)
\end{aligned}
$$

onde $\gamma=\left(1-v^{2} / c^{2}\right)^{-\frac{1}{2}}$.

Tendo em vista a necessidade de se formular leis físicas invariantes por transformações do tipo (1), uma quantidade de grande importância é o intervalo infinitesimal entre dois eventos, definido por

$$
d s^{2}=c^{2} d t^{2}-d x^{2}-d y^{2}-d z^{2} .
$$

Como pode ser visto $d s$, assim como o intervalo finito $\Delta s$, se mantém inalterado quando escrito em termos das coordenadas do referencial $K^{\prime}$ pelas transformações em (1). Ou seja, $d s^{\prime 2}=d s^{2}$. Diz-se, então, que o intervalo entre dois eventos é invariante por transformações de Lorentz. Ou também, que $d s$ é um escalar de Lorentz [2].

Como se vê, na relatividade especial a quantidade invariante, similar à distância entre dois pontos no espaço euclidiano, é uma combinação de tempo e espaço definida em (2). Então, para descrever as leis físicas dentro do arcabouço da relatividade especial torna-se conveniente a utilização de um espaçotempo quadridimensional com "distância" invariante $d s$ : o espaço de Minkowski.

No espaço de Minkowski as coordenadas $(c t, x, y, z)$ de um evento podem ser vistas como as componentes de um quadrivetor contravariante $x^{\mu}=\left(x^{0}, x^{1}, x^{2}, x^{3}\right)$, com $x^{0}=c t, x^{1}=x, x^{2}=y$ e $x^{3}=z$, que sob mudança de referencial transforma-se acordando a [2]

$$
x^{\prime \mu}=\Lambda_{\nu}^{\mu} x^{\nu},
$$

onde $\Lambda_{\nu}^{\mu}$ é a matriz da transformação de Lorentz ${ }^{1}$ No caso particular da transformação (1), a matriz em questão é

$$
\left(\Lambda^{\mu}{ }_{\nu}\right)=\left(\begin{array}{cccc}
\gamma & -\frac{v}{c} \gamma & 0 & 0 \\
-\frac{v}{c} \gamma & \gamma & 0 & 0 \\
0 & 0 & 1 & 0 \\
0 & 0 & 0 & 1
\end{array}\right)
$$

\footnotetext{
${ }^{1}$ Neste trabalho é adotada a notação de Einstein onde índices repetidos representam um somatório, tal que $\nu$ em (3) é somado de 0 a 3.
}

Em suma, um quadrivetor contravariante é um conjunto de quatro quantidades $A^{0}, A^{1}, A^{2}$ e $A^{3}$, que se transformam respectivamente da mesma maneira que as componentes $x^{0}, x^{1}, x^{2}$ e $x^{3}$ de $x^{\mu}$, frente a uma transformação de Lorentz [2,3].

A introdução de um tensor métrico com componentes $\eta_{\mu \nu}=\operatorname{diag}(1,-1,-1,-1)$, conhecido como a métrica de Minkowski, permite escrever o quadrado da magnitude de um quadrivetor contravariante na forma

$$
\eta_{\mu \nu} x^{\mu} x^{\nu}=\left(x^{0}\right)^{2}-\left(x^{1}\right)^{2}-\left(x^{2}\right)^{2}-\left(x^{3}\right)^{2},
$$

e essa quantidade caracteriza um escalar de Lorentz. Em particular, $d s^{2}=\eta_{\mu \nu} d x^{\mu} d x^{\nu}$.

Do resultado (4) define-se o abaixamento de índice, $x_{\mu}=\eta_{\mu \nu} x^{\nu}$, tal que o produto escalar de dois quadrivetores contravariantes quaisquer, $x^{\mu}$ e $y^{\mu}$ por exemplo, é dado por

$$
\eta_{\mu \nu} x^{\mu} y^{\nu}=x_{\nu} y^{\nu}
$$

E, em especial, a magnitude quadrada de um quadrivetor $x^{\mu}$ é $\eta_{\mu \nu} x^{\mu} x^{\nu}=x^{\mu} x_{\mu}[3]$.

De maneira similar, introduz-se o tensor métrico inverso $\eta^{\mu \nu}$ que possui as mesmas componentes de $\eta_{\mu \nu}$, tal que $\eta^{\mu \alpha} \eta_{\alpha \nu}=\delta_{\nu}^{\mu}$, onde $\delta_{\nu}^{\mu}$ é o delta de Kronecker [2,3]. A partir da métrica inversa o levantamento de índice é definido como $x^{\mu}=\eta^{\mu \nu} x_{\nu}$.

Uma vez que o levantamento ou abaixamento de índice relaciona $x_{\mu}$ a $x^{\mu}$ ou vice-versa, a lei de transformação de $x_{\mu}$ pode ser determinada a partir de (3), como segue:

$$
x^{\prime \mu}=\Lambda_{\alpha}^{\mu} x^{\alpha}=\Lambda_{\alpha}^{\mu} \eta^{\alpha \beta} x_{\beta},
$$

multiplicando à esquerda por $\eta_{\gamma \mu}$, vem

$$
x_{\gamma}^{\prime}=\eta_{\gamma \mu} \Lambda_{\alpha}^{\mu} \eta^{\alpha \beta} x_{\beta} .
$$

Agora, definindo $\eta_{\gamma \mu} \Lambda_{\alpha}^{\mu} \eta^{\alpha \beta}=\Lambda_{\gamma}{ }^{\beta}$, tem-se

$$
x_{\gamma}^{\prime}=\Lambda_{\gamma}^{\beta} x_{\beta} \text {. }
$$

É possível mostrar, pela invariância do produto escalar definido em (5), que $\Lambda_{\mu}{ }^{\nu} \Lambda^{\mu}{ }_{\alpha}=\delta_{\alpha}^{\nu}[2]$. As quatro quantidades $x_{\mu}$ juntamente com a lei de transformação (8) definem um quadrivetor covariante no espaço de Minkowski. O protótipo de um quadrivetor covariante é o gradiente de uma função escalar [1-3]

$$
\partial_{\mu} \phi=\frac{\partial \phi}{\partial x^{\mu}}=\left(\frac{\partial \phi}{\partial x^{0}}, \frac{\partial \phi}{\partial x^{j}}\right)
$$


onde $j=1,2,3$ enumera as coordenadas espaciais tridimensionais $x, y, z$. Aqui, vale ressaltar que uma função escalar $\phi\left(x^{\mu}\right)$ é aquela cujo valor em cada ponto do espaço-tempo é invariante por transformações de Lorentz [2]

$$
\phi\left(x^{\mu}\right)=\phi^{\prime}\left(x^{\prime \mu}\right) .
$$

Uma quantidade de grande importância na relatividade especial é o intervalo de tempo próprio $d \tau$, definido como o intervalo de tempo medido pelo referencial que acompanha um determinado objeto 3 . Nesse referencial, $K^{\prime}$ por exemplo, o intervalo invariante medido é $d s^{\prime 2}=c^{2} d \tau^{2}$, pois as coordenadas espaciais são fixas. No entanto, para o referencial $K$, que vê $K^{\prime}$ se mover com velocidade constante $v$, o intervalo invariante é

$$
d s^{2}=c^{2} d t^{2}-d x^{2}-d y^{2}-d z^{2}=c^{2} d t^{2}\left(1-v^{2} / c^{2}\right) .
$$

Como $d s^{\prime 2}=d s^{2}$, então

$$
d \tau=d s / c=d t / \gamma
$$

Deve ser notado que $d \tau$, ao contrário de $d t$, é um escalar de Lorentz. Assim, o intervalo de tempo próprio torna-se muito relevante para a formulação quadridimensional das leis físicas.

O espaço de Minkowski descrito aqui é de grande utilidade quando se pretende formular as leis físicas no contexto da relatividade especial. Como um exemplo disso no restante deste trabalho faz-se uso desse espaço na busca por um modelo de gravitação relativística.

\section{Gravitação escalar}

Uma teoria de gravitação que esteja em acordo com a relatividade especial deve levar a novas previsões que possam ser verificadas experimentalmente ao mesmo tempo em que, no limite de baixas velocidades e campo estático, deve recuperar os resultados para o campo gravitacional de Newton.

O ponto de partida para uma teoria de gravitação escalar é a ação para a partícula de massa $m$ na presença do campo $\phi\left(x^{\mu}\right)$ dada por ${ }^{2}$

$$
S=-\int_{\tau 1}^{\tau 2}\left(m c+\frac{\lambda}{c} \phi\right) d s
$$

onde $d s=\sqrt{\eta_{\mu \nu} d x^{\mu} d x^{\nu}}=c d \tau$ é o elemento de linha no espaço de Minkowski e $\lambda$ é uma constante que

\footnotetext{
${ }^{2}$ Por simplicidade adota-se $\phi\left(x^{\mu}\right)=\phi$ daqui por diante.
}

contém informação acerca da "carga" da partícula. Assim, na ação anterior o primeiro termo descreve a partícula livre enquanto o segundo descreve o acoplamento entre a partícula e o campo.

Aplicando o princípio da mínima ação de Hamilton na variação de 13$)$ com respeito a $x^{\mu}$ chega-se às equações de movimento,

$$
\frac{d}{d \tau}\left[\left(m+\frac{\lambda}{c^{2}} \phi\right) U^{\mu}\right]=\lambda \eta^{\mu \nu} \partial_{\nu} \phi
$$

onde $U^{\mu}=\frac{d x^{\mu}}{d \tau}=\gamma(c, \mathbf{v})$ é o quadrivetor velocidade da partícula [2,3. No limite de baixas velocidades e campo estático, no qual, respectivamente, $c \rightarrow$ $\infty$ e $\partial_{0} \phi \ll \partial_{j} \phi \operatorname{com} j=1,2,3$, as equações de movimento (14) tornam-se

$$
m \frac{d \mathbf{v}}{d t}=-\lambda \boldsymbol{\nabla} \phi,
$$

onde $\boldsymbol{\nabla} \phi$ é o gradiente de $\phi$. Como $\mathbf{v}$ é a velocidade tridimensional da partícula a dinâmica torna-se semelhante à de Newton nesse limite, levando, inclusive, ao próprio resultado newtoniano se o produto $\lambda \phi$ for análogo à energia potencial gravitacional no limite em questão.

A dinâmica do campo é dada adicionando-se um termo em (13) que depende apenas das derivadas de $\phi$, tal que $S$ toma a forma

$$
S=-m c \int_{\tau_{1}}^{\tau_{2}} d s-\frac{\lambda}{c} \int_{\tau_{1}}^{\tau_{2}} \phi d s+\frac{1}{2} \int \eta^{\mu \nu} \phi_{, \mu} \phi_{, \nu} d^{4} x
$$

Nesta ação o campo $\phi$ é visto como uma variável dinâmica de forma que a variação de $S$ com respeito a $\phi$ leva às equações de movimento que determinam o campo. Antes de realizar essa variação é conveniente escrever o segundo membro de (16) como uma integral em $d^{4} x$, similarmente ao último membro. Para efetuar essa tarefa faz-se uso de uma função delta de Dirac em quatro dimensões.

Em uma dimensão a função delta de Dirac, $\delta(x-$ $z$ ), é definida pelas propriedades [4]:

$$
\delta(x-z)=0 \quad \text { para todo } \quad x \neq z
$$

e

$$
\int_{-\infty}^{+\infty} \delta(x-z) d x=1
$$

onde $z$ é um ponto qualquer. Essa função pode ser estendida para espaços com um número $n$ de dimensões, e uma função delta nesses espaços é dada pelo produto de $n$ funções delta, uma para cada 
dimensão do espaço em questão 4. Em particular, no espaço quadridimensional

$$
\delta^{(4)}\left[x^{\mu}\right]=\delta\left(x^{0}\right) \delta\left(x^{1}\right) \delta\left(x^{2}\right) \delta\left(x^{3}\right) .
$$

Assim, 17 e 18 podem ser generalizadas, por exemplo, para quatro dimensões:

$$
\delta^{(4)}\left[x^{\mu}-z^{\mu}\right]=0 \quad \text { para todo } \quad x^{\mu} \neq z^{\mu}
$$

e

$$
\int_{-\infty}^{+\infty} \ldots \int_{-\infty}^{+\infty} \delta^{(4)}\left[x^{\mu}-z^{\mu}\right] d x^{0} \ldots d x^{3}=1 .
$$

Vale salientar que não é necessário estender o intervalo de integração de $-\infty$ a $+\infty$, ele apenas deve conter o ponto $x=z$, ou $x^{\mu}=z^{\mu}$ no caso quadridimensional.

A partir das definições da função delta de Dirac segue uma propriedade de grande relevância aqui, válida para qualquer função $f\left(x^{\mu}\right)$ bem comportada:

$$
\int f\left(x^{\mu}\right) \delta^{(4)}\left[x^{\mu}-z^{\mu}\right] d^{4} x=f\left(z^{\mu}\right) .
$$

Essa propriedade permite escrever o segundo termo em (16) para uma partícula seguindo a trajetória $z^{\mu}=z^{\mu}(\tau)$ na forma

$$
\int_{\tau} \phi\left(z^{\mu}\right) d s=\int_{\tau}\left[\int_{R} \phi\left(x^{\mu}\right) \delta^{(4)}\left[x^{\mu}-z^{\mu}\right] d^{4} x\right] d s .
$$

A integral em $d^{4} x$ é tomada sobre uma região $R$ do espaço-tempo, enquanto a integral em $d s$ é tomada ao longo da trajetória da partícula. Logo, pode-se escrever

$$
\int_{\tau} \phi\left(z^{\mu}\right) d s=\int_{R}\left[\int_{\tau} \delta^{(4)}\left[x^{\mu}-z^{\mu}\right] d s\right] \phi\left(x^{\mu}\right) d^{4} x .
$$

A expressão entre colchetes na última igualdade é a "densidade de partícula" $\rho\left(x^{\mu}\right)$ para uma partícula pontual seguindo a trajetória $z^{\mu}=z^{\mu}(\tau)$ [1]. A $\delta^{(4)}\left[x^{\mu}-z^{\mu}(\tau)\right]$ deixa evidente que a densidade é nula em todo o espaço, exceto sobre a trajetória da partícula.

Agora, por meio de $\rho\left(x^{\mu}\right)$, o segundo termo da ação 16 é reescrito como

$$
-\frac{\lambda}{c} \int \phi d s=-\frac{\lambda}{c} \int \rho\left(x^{\mu}\right) \phi\left(x^{\mu}\right) d^{4} x
$$

e a variação com respeito a $\phi$ pode ser realizada. Lembrando que o primeiro termo de $S$ é nulo para essa variação, chega-se à equação de campo na presença de font $\S^{3}$

$$
\square \phi=-\frac{\lambda}{c} \rho,
$$

que no limite newtoniano, $\phi_{, 0} \ll \phi_{, j}$, torna-se

$$
\nabla^{2} \phi=\frac{\lambda}{c} \rho
$$

Uma vez que $\phi$ descreve o campo gravitacional na relatividade especial, no limite considerado (27) deve recuperar a equação de Poisson para a gravitação de Newton [5]

$$
\nabla^{2} \phi_{N}=4 \pi G \rho_{m}
$$

sendo $\rho_{m}$ a densidade de massa, $G$ a constante gravitacional e $\phi_{N}$ o potencial newtoniano.

Desde que o campo $\phi$ interage com a matéria onde "carga" é a massa, a constante $\lambda$ deve ser proporcional a $m$, o que torna conveniente a definição das novas variáveis $\kappa m=\lambda, \Phi=\kappa \phi$ e $\rho_{m}=m \frac{\rho}{c}$. A equação (27) pode ser reescrita em termos dessas variáveis e o resultado $(28)$ é assegurado no limite newtoniano para $\kappa=\sqrt{4 \pi G}[6]$.

Com as novas variáveis a ação 13 toma a forma

$$
S=-m c^{2} \int_{\tau_{1}}^{\tau_{2}}\left(1+\frac{\Phi}{c^{2}}\right) d \tau
$$

na qual é possível notar características favoráveis à gravitação escalar:

- é invariante de Lorentz;

- leva a uma força sempre atrativa [1;

- como a ação tem dimensão de energia vezes tempo, $\Phi$ tem dimensão de velocidade quadrada assim como $\phi_{N}$;

- como a massa é uma constante multiplicativa, as equações de movimento não dependem explicitamente da mesma. Isto representa, aqui, o análogo ao princípio da equivalência newtoniano;

- é uma teoria de gravitação linear, no sentido de que o campo produzido pela soma de duas fontes é igual à soma dos dois campos gerados independentemente por cada uma delas 7 .

É interessante notar, ainda, que escrevendo 26. em termos das novas variáveis e substituindo o valor de $\kappa$ anterior, tem-se

$$
\square \Phi=-4 \pi G \rho_{m},
$$

\footnotetext{
$\overline{{ }^{3} \square \phi=\eta^{\mu \nu} \partial_{\mu} \partial_{\nu} \phi=\partial_{\mu} \partial^{\mu}} \phi$ é o operador de d'Alembert.
} 
que pode ser vista como a "Equação de Einstein" para a gravitação escalar. Essa equação representa a generalização mais simples da equação de Poisson (28) na tentativa de descrever o campo gravitacional no contexto relativístico e foi inicialmente tratada por Nordström 8 .

A teoria até aqui apresentada carece de uma generalização a mais. Como pode ser visto a única e exclusiva fonte do campo gravitacional nas equações anteriores é a densidade de massa $\rho_{m}$. No entanto, como na relatividade especial massa e energia se equivalem, isso restringe a aplicação da teoria aos casos em que a fonte do campo é um único sistema de partículas. Porque, agora, o campo produzido por um conjunto de fontes deve levar em conta todo o conteúdo de energia existente, inclusive, a energia de interação entre as fontes [7]. Logo, $\rho_{m}$ deve ser generalizada de tal modo a incluir como fonte do campo gravitacional qualquer conteúdo de energia presente no sistema físico sob consideração.

A informação sobre o conteúdo de energia e momento de um sistema físico e as leis de conservação dessas quantidades podem ser expressas em forma quadridimensional por meio do tensor energia-momento definido como 3

$$
T^{\mu \nu}=-\eta^{\mu \nu} \mathcal{L}+\sum_{j} \frac{\partial \mathcal{L}}{\partial\left(\partial_{\mu} q^{(j)}\right)} \partial^{\nu} q^{(j)},
$$

onde os $q^{(j)}$ 's são quaisquer variáveis dinâmicas descrevendo o sistema e $\mathcal{L}=\mathcal{L}(q, q, \mu)$ é a densidade lagrangiana advinda da ação,

$$
S=\int \mathcal{L} d^{4} x
$$

A fonte do campo gravitacional pode ser generalizada por meio de um escalar de Lorentz construído a partir do tensor energia-momento: o traço de $T^{\mu \nu}$, dado por

$$
T=\eta_{\mu \nu} T^{\mu \nu} .
$$

Em especial, para um sistema de partículas pontuais à pressão nula essa quantidade é conhecida ser

$$
T=\rho_{m} c^{2},
$$

com $\rho_{m}$ a densidade de massa das partículas. Assim, a parte da ação (16) que descreve $\Phi$ pode ser reescrita contendo $T$ como a fonte do campo,

$$
S=\int\left(-\frac{\Phi T}{c^{2}}+\frac{1}{8 \pi G} \eta^{\mu \nu} \Phi_{, \nu} \Phi_{, \mu}\right) d^{4} x
$$

Da mesma forma a equação de campo na presença de fonte torna-se

$$
\square \Phi=-\frac{4 \pi G}{c^{2}} T .
$$

Generalizada a fonte do campo surge uma questão de grande relevância a ser tratada na gravitação escalar. Pode-se notar, levando o último termo de (35) em (31), que o traço do tensor energia-momento associado ao campo $\Phi$ é diferente de zero. Assim, $T$ aparecendo nas equações anteriores leva em conta a contribuição de todas as fontes para o campo gravitacional, exceto o próprio campo gravitacional.

A solução para essa questão seria acoplar o campo gravitacional ao traço do seu próprio tensor energiamomento. Em princípio isso pode ser feito e uma das consequências do resultado é que a densidade lagrangiana inicial ganha um potencial $V(\Phi)$ pela adição de termos dependentes do campo (veja, por exemplo, [1,8]). A presença de $V(\Phi)$ devido a autointeração quebra a linearidade, inicialmente afirmada para a teoria de gravitação escalar, pelo fato que a equação de campo na presença de fonte deixa de ser uma equação linear.

Uma vez que a teoria é não-linear o princípio da superposição, característico da gravitação newtoniana, deixa de ser válido. Um efeito imediato disso é que se $\Phi_{1}$ e $\Phi_{2}$ são os campos produzidos de forma isolada pelas respectivas fontes $\rho_{1}$ e $\rho_{2}$, o campo $\Phi_{1+2}$ produzido quando essas duas fontes são somadas é diferente da soma dos campos $\Phi_{1} \mathrm{e}$ $\Phi_{2}$, gerados individualmente. Traduzindo em linguagem matemática, $\Phi\left(\rho_{1}+\rho_{2}\right) \neq \Phi\left(\rho_{1}\right)+\Phi\left(\rho_{2}\right)$. No entanto, a não-linearidade da teoria evidencia o fato, anteriormente mencionado, de que na relatividade especial a equivalência entre massa e energia faz com que o novo campo, gerado pela soma de fontes antes independentes, leve em conta a energia de interação gravitacional entre elas. Ou seja, a própria gravidade é vista como fonte de mais gravidade 7 .

Vale ressaltar que a não-linearidade também está presente na teoria da relatividade geral de Einstein e que em certos casos é o ingrediente chave das implicações dessa teoria 7 .

\section{4. Órbitas planetárias}

Como um teste de verificação da gravitação escalar os resultados da seção precedente são aplicados ao estudo do movimento planetário dentro da proposta do exercício 3.1 da Ref. [1]. 
A ação (29) pode ser vista como uma imediata generalização da ação para a partícula livre, na qual o intervalo de linha $d s^{2}=\eta_{\mu \nu} d x^{\mu} d x^{\nu}$ é substituído por $d s_{\Phi}^{2}=g_{\mu \nu} d x^{\mu} d x^{\nu}$, onde

$$
g_{\mu \nu}=\left(1+\frac{\Phi}{c^{2}}\right)^{2} \eta_{\mu \nu}
$$

Assim, a ação para uma partícula de massa $m$ na presença do campo $\Phi$ torna-se

$$
S=-m c \int_{\tau_{1}}^{\tau_{2}} d s_{\Phi}=-m c \int_{\tau_{1}}^{\tau_{2}} \sqrt{g_{\mu \nu} d x^{\mu} d x^{\nu}}
$$

que leva à equação de Hamilton-Jacobi [1, $, 3,6]$

$$
g^{\mu \nu} S_{, \mu} S_{, \nu}=m^{2} c^{2}
$$

com $g^{\mu \nu}$ a inversa de (37).

No estudo do movimento planetário a densidade $\rho_{m}$ é considerada devida a uma massa $M$ fixa na origem do sistema de coordenadas e a equação (30) é tomada no limite de baixas velocidades e campo estático. Logo, a equação de campo é a usual equação de Poisson para o potencial gravitacional newtoniano tendo como solução

$$
\Phi(r)=-\frac{G M}{r},
$$

onde $r=\|\mathbf{r}\|$, com $\mathbf{r}=(x, y, z)$ o vetor que localiza $m$ a partir da origem.

A solução para $\Phi$ na forma 40 configura o sistema sob descrição como o movimento de uma partícula de massa $m$ na presença de um campo de força central cuja energia potencial só depende da distância entre $m$ e a massa $M$ que produz o campo, sem levar em conta a orientação. Dessa forma o sistema possui simetria esférica e as equações de movimento devem ser invariantes por rotação em torno de qualquer eixo fixo que passe pela origem das coordenadas. Tal invariância é garantida pela conservação do vetor momento angular o que restringe o movimento da partícula a um plano perpendicular a esse vetor [5].

Com as considerações anteriores a equação (39) pode ser escrita explicitamente em termos das coordenadas polares planas $(r, \varphi)$ na forma

$$
\begin{array}{r}
\left(1+\frac{\Phi}{c^{2}}\right)^{-2}\left[\frac{1}{c^{2}}\left(\frac{\partial S}{\partial t}\right)^{2}-\left(\frac{\partial S}{\partial r}\right)^{2}\right. \\
\left.-\frac{1}{r^{2}}\left(\frac{\partial S}{\partial \varphi}\right)^{2}\right]-m^{2} c^{2}=0
\end{array}
$$

o que é idêntico a escrevê-la nas coordenadas esféricas $(r, \theta, \varphi)$ e restringir o movimento ao plano $\theta=$ $\frac{\pi}{2}$. Uma solução para essa equação pode ser proposta notando-se que a energia do sistema sob consideração, dada pela hamiltoniana associada a (29), é

$$
E=\frac{m c^{2}+m \Phi}{\sqrt{1-\frac{v^{2}}{c^{2}}}}
$$

a qual no limite não relativístico torna-se

$$
E=m c^{2}+\frac{1}{2 m}\left(p_{r}^{2}+\frac{p_{\varphi}^{2}}{r^{2}}\right)+m \Phi=m c^{2}+\mathcal{E}
$$

onde $p_{r}$ e $p_{\varphi}$ são os momentos conjugados a $r$ e $\varphi$, respectivamente, e $\mathcal{E}$ é a energia na aproximação newtoniana. Como pode ser visto a função hamiltoniana não depende explicitamente do tempo, tal que a energia $E$ é conservada. Levando-se em conta que o momento angular $L$ também é conservado, a equação (41) admite uma solução por separação de variáveis na forma 1,2]

$$
S=-E t+L \varphi+S_{r},
$$

onde $S_{r}$ é a parte da solução que depende apenas da trajetória.

Substituindo a solução anterior em (41) tem-se

$$
\left(\frac{d S_{r}}{d r}\right)^{2}=\frac{E^{2}}{c^{2}}-\frac{L^{2}}{r^{2}}-m^{2} c^{2}\left(1+\frac{2 \Phi}{c^{2}}+\frac{\Phi^{2}}{c^{4}}\right)
$$

que reescrita na forma integral, com $\Phi$ dado por (40), leva a

$$
S_{r}=\int d r\left[\frac{E^{2}}{c^{2}}-m^{2} c^{2}+\frac{2 k m}{r}-\frac{1}{r^{2}}\left(L^{2}+\frac{k^{2}}{c^{2}}\right)\right]^{\frac{1}{2}},
$$

com $k=G M m$.

Para estudar a trajetória $r$ como função do ângulo $\varphi$ é conveniente tomar

$$
\frac{\partial S}{\partial L}=\varphi_{0}=\text { constante }
$$

o que, pela substituição em (44), fornece

$$
\frac{\partial S}{\partial L}=\varphi+\frac{\partial S_{r}}{\partial L}=\varphi_{0} .
$$

Derivando $S_{r}$ com respeito a $L$ e usando a condição anterior, chega-se a

$$
\begin{aligned}
\varphi-\varphi_{0}=\int & d r\left[\frac{E^{2}}{c^{2}}-m^{2} c^{2}+\frac{2 k m}{r}\right. \\
& \left.-\frac{1}{r^{2}}\left(L^{2}+\frac{k^{2}}{c^{2}}\right)\right]^{-\frac{1}{2}} \frac{L}{r^{2}},
\end{aligned}
$$


que derivada com respeito a $r$ provê

$\frac{d \varphi}{d r}=\left[\frac{E^{2}}{c^{2}}-m^{2} c^{2}+\frac{2 k m}{r}-\frac{1}{r^{2}}\left(L^{2}+\frac{k^{2}}{c^{2}}\right)\right]^{-\frac{1}{2}} \frac{L}{r^{2}}$.

Agora, a equação que determina a trajetória $r=r(\varphi)$ é dada a partir do último resultado por

$$
\begin{array}{r}
\left(\frac{d r}{d \varphi}\right)^{2}=\frac{r^{4}}{L^{2}}\left[\frac{E^{2}}{c^{2}}-m^{2} c^{2}+\frac{2 k m}{r}\right. \\
\left.-\frac{1}{r^{2}}\left(L^{2}+\frac{k^{2}}{c^{2}}\right)\right] .
\end{array}
$$

Para estudar a última equação introduz-se a nova variável $u=\frac{1}{r}$, tal que

$$
\frac{d u}{d \varphi}=\frac{d u}{d r} \frac{d r}{d \varphi}=-\frac{1}{r^{2}} \frac{d r}{d \varphi} \quad \Longrightarrow \quad \frac{d r}{d \varphi}=-r^{2} \frac{d u}{d \varphi} .
$$

Pela última igualdade, (51) pode ser reescrita em termos de $u$ e o resultado, depois de derivado com respeito a $\varphi$, leva a

$\frac{d^{2} u}{d \varphi^{2}}+\omega^{2} u=\frac{k m}{L^{2}}, \quad$ onde $\quad \omega^{2}:=\left(1+\frac{k^{2}}{L^{2} c^{2}}\right)$.

Esta é a equação de um oscilador harmônico sob ação de uma força constante e pode ser completamente solucionada dada as condições iniciais.

No problema em questão, considerando-se apenas o caso de movimento confinado onde $E<m c^{2}$, as condições iniciais para (53) são obtidas a partir dos pontos de "retorno" da trajetória, para os quais a velocidade radial $\frac{d r}{d t}$ é nula. Como

$$
\begin{array}{r}
\frac{d r}{d t}=-\frac{1}{u^{2}} \frac{d u}{d t}=-\frac{1}{u^{2}} \frac{d u}{d \varphi} \frac{d \varphi}{d t}= \\
-r^{2} \frac{d \varphi}{d t} \frac{d u}{d \varphi}=-\frac{L}{m} \frac{d u}{d \varphi}
\end{array}
$$

esses pontos podem também serem determinados através de $\frac{d u}{d \varphi}=0 \mathrm{ou}$, como assegurado a partir de $\left(52\right.$, por $\frac{d r}{d \varphi}=0$. Com isto os pontos de "retorno" ficam estabelecidos pelo resultado (51) por meio de

$$
E^{2}-m^{2} c^{4}+\frac{2 k m c^{2}}{r}-\frac{L^{2} \omega^{2} c^{2}}{r^{2}}=0 .
$$

A equação anterior é quadrática em $\frac{1}{r}$ com soluções

$$
\frac{1}{r}=\frac{k m}{L^{2} \omega^{2}} \pm \sqrt{\left(\frac{k m}{L^{2} \omega^{2}}\right)^{2}-\frac{m^{2} c^{4}-E^{2}}{L^{2} \omega^{2} c^{2}}},
$$

onde o sinal positivo representa o menor valor de $r$ e consequentemente o ponto de maior aproximação da partícula ao centro da força.

Usando um sistema de coordenadas polares adequado pode-se impor que a direção de maior aproximação ocorra em $\varphi=0$ e que nesse ponto $\frac{d u}{d \varphi}=0$. Levando essas duas condições na solução geral da equação (53) dada por

$$
u=A \cos (\omega \varphi)+B \sin (\omega \varphi)+\frac{k m}{L^{2} \omega^{2}},
$$

determina-se as constantes $A$ e $B$ como segue:

$$
\begin{gathered}
u(\varphi=0)=A+\frac{k m}{L^{2} \omega^{2}}=\frac{k m}{L^{2} \omega^{2}} \\
+\sqrt{\left(\frac{k m}{L^{2} \omega^{2}}\right)^{2}-\frac{m^{2} c^{4}-E^{2}}{L^{2} \omega^{2} c^{2}}},
\end{gathered}
$$

pois a maior aproximação ocorre em $\varphi=0$ pela escolha do sistema de coordenadas. Assim,

$$
A=\frac{k m}{L^{2} \omega^{2}} \sqrt{1-\frac{\left(m^{2} c^{4}-E^{2}\right) L^{2} \omega^{2}}{k^{2} m^{2} c^{2}}},
$$

enquanto $B$ é determinado por

$$
\frac{d u}{d \varphi}(\varphi=0)=B \omega=0
$$

tal que $B=0$. Dessa forma a solução encontrada para (53) pode ser escrita como

$$
u(\varphi)=\frac{k m}{L^{2} \omega^{2}}(1+\epsilon \cos (\omega \varphi))
$$

onde

$$
\epsilon^{2}=1-\frac{\left(m^{2} c^{4}-E^{2}\right) L^{2} \omega^{2}}{k^{2} m^{2} c^{2}}
$$

é a excentricidade da órbita. Levando em conta a energia não relativística $\mathcal{E}=E-m c^{2}$ (vide Eq. 43 ) e o limite de $c \rightarrow \infty$, tem-se $\omega^{2} \approx 1$ e $\epsilon^{2} \approx 1+\frac{2 \mathcal{E L} L^{2}}{k^{2} m}$, recuperando, assim, o resultado newtoniano padrão 1, 2,9 .

O fato de se ter $\omega \neq 1$ na gravitação escalar significa que os pontos $\varphi=0$ e $\varphi=2 \pi$ são distintos e a órbita não é fechada. Completado o período a partícula não retorna ao ponto inicial e a elipse tem um movimento de precessão. Em particular, desde que $\omega>1$, a trajetória atinge o ponto equivalente a $\varphi=0$ antes mesmo de completar $\varphi=2 \pi$. Diz-se, então, que há um retrocesso. Isso, inclusive, pode ser mensurado impondo

$$
(2 \pi+\triangle \varphi) \omega=2 \pi,
$$




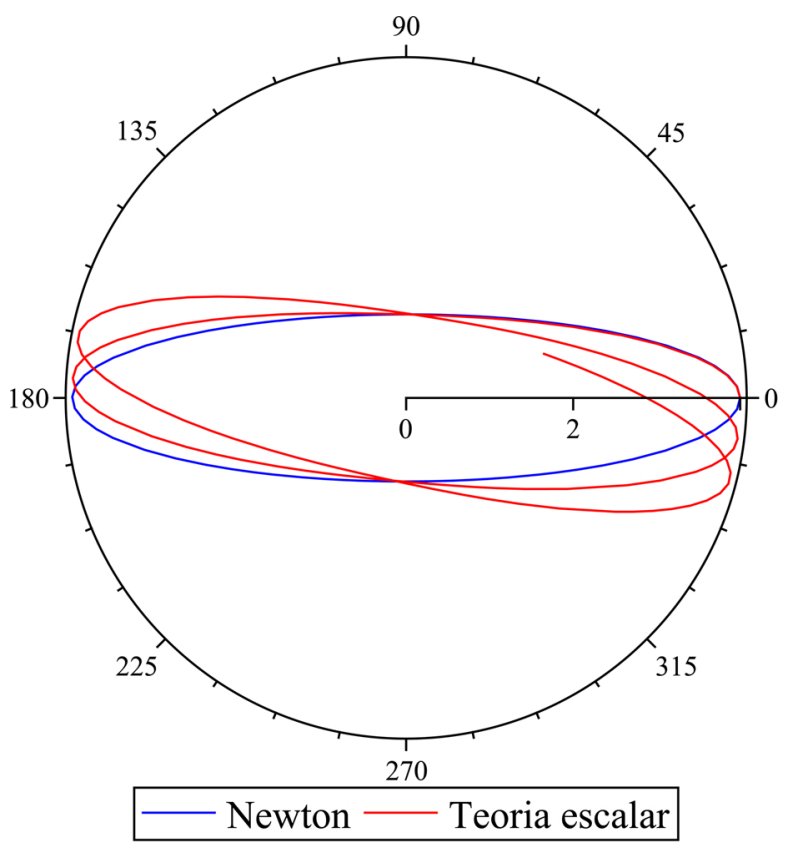

(a)

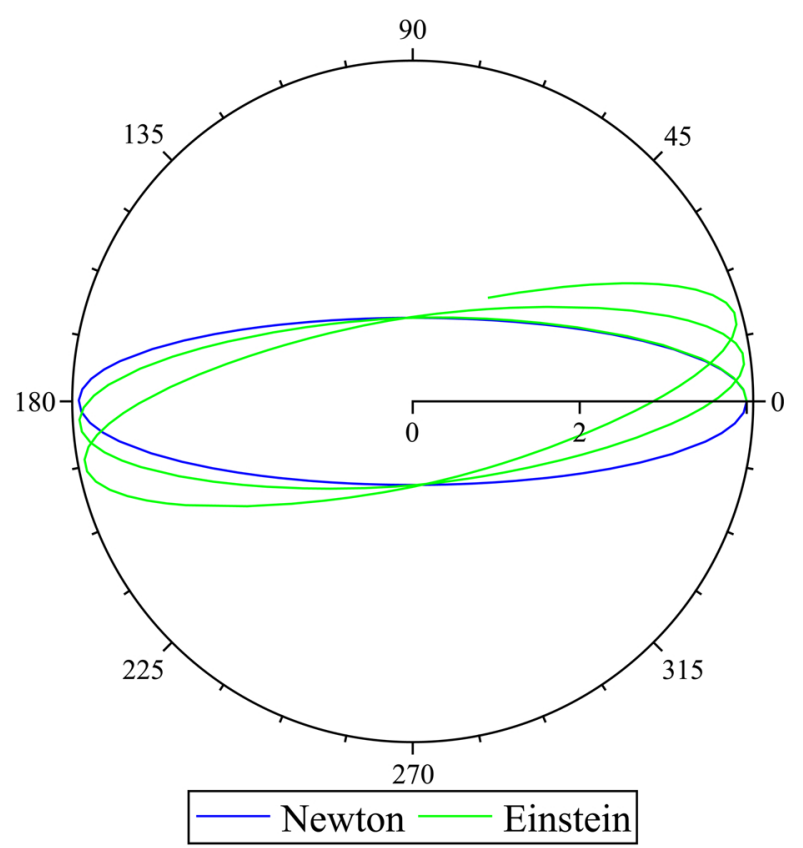

(b)

Figura 1: Em (a) o movimento de retrocesso do periélio $(\varphi=0)$ previsto pela gravitação escalar é apresentado em relação à predição da gravitação de Newton. Já em (b) apresenta-se o real resultado da relatividade geral para o movimento de avanço do periélio em ralação ao caso newtoniano. As figuras (a) e (b) foram confeccionadas a partir de (61) e do resultado de Einstein na literatura [1,10], respectivamente.

o que leva a

$$
\triangle \varphi=2 \pi\left(\omega^{-1}-1\right)
$$

Expandindo $\omega^{-1}$ para $\frac{G^{2} M^{2} m^{2}}{L^{2} c^{2}} \ll 1$, obtem-se

$$
\triangle \varphi \simeq-\frac{G^{2} M^{2} m^{2}}{L^{2} c^{2}} \pi
$$

Como $\varphi=0$ representa o ponto da órbita com maior proximidade entre as massas (periélio), a gravitação escalar prevê um movimento de precessão que é caracterizado pelo retrocesso do periélio a uma taxa $\triangle \varphi$ dada por 65 . Esse resultado está em acordo com o esperado para a solução do exercício 3.1 da Ref. [1], e mostra uma inconsistência entre a previsão da gravitação escalar e os dados experimentais (ver Fig. 1), pois o que realmente se observa é um avanço do periélio, que a relatividade geral de Einstein prevê com grande precisão como sendo $1,5,10$

$$
\triangle \varphi=6 \pi \frac{G^{2} M^{2} m^{2}}{L^{2} c^{2}} .
$$

Ou seja, o resultado 65 é $-\frac{1}{6}$ do resultado previsto por Einstein.

\section{Considerações finais}

Antes de concluir deve ser enfatizado que mesmo apresentando algumas características necessárias para descrever o campo de Newton na relatividade especial, a falha na previsão do movimento de precessão das órbitas planetárias invalida totalmente a gravitação escalar. Deve ser notado também que este modelo de gravitação não será capaz de prever outros fenômenos hoje observados, como é o caso da curvatura dos raios de luz por um campo gravitacional [1. Este fenômeno, em particular, porque o tensor energia-momento do campo eletromagnético tem traço nulo. Assim, a equação 36 não prevê o acoplamento entre a radiação eletromagnética (luz) e o campo gravitacional. Por outro lado, escrever a ação da partícula no campo na forma pode ser visto como uma primeira motivação para se buscar uma íntima ligação entre gravitação e geometria do espaço-tempo.

\section{Agradecimentos}

À CAPES, pelo auxílio financeiro. Ao meu orientador de mestrado, Prof. Dr. Edisom de Souza Moreira 
Junior, pelo incentivo e ensino. Ao árbitro, pelas considerações e sugestões feitas a este trabalho.

\section{Referências}

[1] T. Padmanabhan, Gravitation: Foudations and Frontiers (Cambridge University Press, New York, 2010).

[2] N.A. Lemos, Mecânica Analítica (Editora Livraria da Física, São Paulo, 2007), $2^{\mathrm{a}}$ ed.

[3] L.D. Landau and E.M. Lifshitz, Course of Theoretical Physics, vol. 2, The Classical Theory of Fields (Butterworth-Heinemann, New York, 1994), $4^{\mathrm{a}}$ ed.

[4] R.K. Pathria, Statistical Mechanics (ButterworthHeinemann, Oxford, 1996), $2^{\text {a }}$ ed.

[5] S.T. Thornton and J.B. Marion, Dinâmica Clássica de Partículas e Sistemas (Cengage Learning, São Paulo, 2011).

[6] T.G. Ribeiro, Radiação de Corpo Negro do Campo Escalar. Dissertação de Mestrado, IFQ-UNIFEI, Itajubá (2014), disponível em http://repositorio.unifei.edu.br: 8080/xmlui/bitstream/handle/123456789/ 317/dissertacao_ribeiro2_2014.pdf

[7] M. Pössel, Einstein Online 03, 1002 (2007), disponível em http://www.einstein-online. info/spotlights/gravity_of_gravity, acesso em 2016.

[8] F. Ravndal, arXiv preprint arXiv:gr-qc/0405030 (2004).

[9] K. Watari, Mecânica Clássica, vol. 2 (Editora Livraria da Física, São Paulo, 2003).

[10] J.L. Anderson, Principles of Relativity Physics (Academic Press Inc, New York, 1967). 